\section{Avaliando a difusão de tecnologias médicas no sistema de saúde privado no Brasil: o caso da tomografia por emissão de pósitrons (PET)}

\section{Assessing the use of medical technology in the private health system in Brazil: the case of Positron Emission Tomography (PET)}

Ana Luiza d'Ávila Viana 1 Hudson Pacífico da Silva 2
1 Departamento de Medicina Preventiva. Faculdade de Medicina. Universidade de São Paulo. Avenida Doutor Arnaldo, 455. Cerqueira César. São Paulo, SP, Brasil. CEP 01246-903.

E-mail: anaviana@usp.br

2 Institut de Recherche en Santé Publique. Université de Montréal. 1430 Boulevard du Mont-Royal. Montreal, QC, Canadá.

\begin{abstract}
Objectives: to analyze the determining factors underlying the extent to which positron emission tomography (PET) is used in a selected medical services provider covered by private health insurance plans in Brazil.

Methods: a qualitative case study was undertaken with data collected by means of in-depth interviews $(N=9)$ with representatives of four private health service providers and three health insurance plan operators.

Results: the decisions to acquire the technology under study are taken by managers of hospitals and clinics that adopt a strategy based on excellence in technology. The factors that influence the decision are: a history of being pioneers in the introduction of new technology; pressure from the institution's clinical staff; the prestige of the physician or the area covered by the unit; the financial resources available; ease of access to technology; competition among health service providers; scientific evidence; and the expected return on the investment.

Conclusions: the policy instruments currently used to manage the use of medical technology in the Brazilian health system has little influence on the decisions made by large-scale private service providers, whose activities are not necessarily related to the health needs of the population or the priorities of public health policy.
\end{abstract}

Key words Diffusion of innovation, Biomedical technology, Positron-emission tomography, Health sciences, technology and innovation management, Brazil

\section{Resumo}

Objetivos: analisar os determinantes da difusão da tomografia por emissão de pósitrons (PET) em um conjunto selecionado de prestadores de serviços e operadoras de planos de saúde no Brasil.

Métodos: estudo de caso qualitativo, com coleta de dados realizada por meio de entrevistas em profundidade $(N=9)$ com representantes de quatro prestadores de serviços de saúde privados e três operadoras de planos de saúde.

Resultados: as decisões para aquisição da tecnologia estudada são tomadas por dirigentes de hospitais e clínicas que adotam uma estratégia de diferenciação baseada em liderança tecnológica. Os fatores que influenciam essa decisão são: histórico de pioneirismo da instituição na incorporação de tecnologias; pressão do corpo clínico vinculado à instituição; prestígio do médico ou da área demandante; disponibilidade de recursos financeiros; acesso facilitado à tecnologia; concorrência entre os prestadores de serviços de saúde; evidências científicas; e rentabilidade do investimento.

Conclusões: os atuais instrumentos de política usados para gerenciar a difusão de tecnologias médicas no sistema de saúde brasileiro exercem pouca influência nas decisões de grandes prestadores privados, cujas atividades não estão necessariamente relacionadas com as necessidades de saúde da população ou com as prioridades da política de saúde.

Palavras-chave Difusão de inovação, Tecnologia biomédica, Tomografia por emissão de pósitrons, Gestão de ciência, tecnologia e inovação em saúde, Brasil 


\section{Introdução}

Em novembro de 2009, o Ministério da Saúde instituiu oficialmente a Política Nacional de Gestão de Tecnologias em Saúde no Brasil, depois de mais de três anos em que a primeira versão da referida política foi submetida à consulta pública.1,2 Entendida "como o conjunto de atividades gestoras relacionadas com os processos de avaliação, incorporação, difusão, gerenciamento da utilização e retirada de tecnologias do sistema de saúde", 1 a gestão de tecnologias em saúde representa um desafio para os sistemas de saúde em todo o mundo, na medida em que as inovações tecnológicas desempenham um papel central na organização do sistema, influenciando a forma como os serviços de saúde são prestados, assim como seus resultados. ${ }^{3}$ Além disso, o número de novas tecnologias em saúde lançadas no mercado é crescente, seu ciclo de vida está diminuindo e o ritmo das mudanças está desafiando a capacidade dos gestores de decidir sobre a incorporação dessas tecnologias nos serviços de saúde. 4

Dois meses mais tarde, em janeiro de 2010, a Agência Nacional de Saúde Suplementar (ANS) publicou a Resolução Normativa 211, que atualiza seu rol de procedimentos e eventos em saúde. $5 \mathrm{~A}$ nova versão desse importante instrumento de política pública, que lista a cobertura mínima obrigatória a ser oferecida pelas operadoras de planos privados de assistência à saúde, inclui nada menos que 70 novos procedimentos médicos e odontológicos, ampliando a cobertura assistencial oferecida aos beneficiários de planos de saúde contratados a partir de 2 de janeiro de 1999, que é a data de entrada em vigor da legislação que regulamenta o setor de saúde suplementar. Entre os novos procedimentos incluídos, está a tomografia por emissão de pósitrons (PET), tecnologia diagnóstica que faz uso de substâncias radioativas para detectar alterações metabólicas de órgãos e tecidos, com aplicações importantes nas áreas de oncologia, neurologia e cardiologia. 6

O que esses dois fatos têm em comum é que ambos são expressões do mesmo fenômeno - a difusão de inovações tecnológicas nos sistemas de saúde. De modo geral, as tecnologias em saúde incluem os métodos e intervenções usadas na promoção da saúde, na prevenção, diagnóstico e tratamento de doenças e na reabilitação de suas consequências e cuidados de longo prazo. Dessa forma, as tecnologias em saúde incluem medicamentos, dispositivos, equipamentos, materiais, agentes diagnósticos, procedimentos médicos e cirúrgicos, assim como processos e sistemas organizacionais e de informação usados na prestação de serviços de saúde. ${ }^{7}$ A difusão de tecnologias, por sua vez, refere-se ao "o processo por meio do qual uma inovação é comunicada através de certos canais ao longo do tempo entre os membros de um sistema social". ${ }^{8}$ Apesar da aparente simplicidade dessas definições, a gestão ou controle da difusão de tecnologias em saúde é uma tarefa complexa. Essa complexidade está relacionada com dois aspectos importantes: de um lado, com a forma por meio da qual as tecnologias são difundidas no meio social em geral, ou seja, com a natureza da difusão tecnológica; de outro, com o contexto das tecnologias em saúde em particular, isto é, as características específicas das tecnologias e dos sistemas de saúde. ${ }^{4}$

A literatura sociológica sobre inovação identifica pelo menos dez fatores críticos que influenciam o ritmo de difusão das tecnologias, ${ }^{9}$ com destaque para os seguintes aspectos: o grau de vantagem relativa da inovação, em termos de lucratividade econômica, prestígio social e outros benefícios; os canais de comunicação (periódicos, congressos, feiras) utilizados para informar e persuadir os adotantes potenciais a respeito de uma nova tecnologia; as normas, regras e redes sociais, que tendem a exercer influência decisiva sobre o ritmo e extensão de uma inovação; os líderes e formadores de opinião, que estão mais expostos às novas ideias e cuja opinião e comportamento servem de exemplo para seus pares; o grau de compatibilidade de uma inovação com as tecnologias e padrões sociais já existentes; e a infraestrura necessária para viabilizar a adoção e o uso de uma inovação. O peso de cada um desses fatores depende, evidentemente, de cada caso particular.

O modelo desenvolvido por Oh et al.,10 baseado na revisão de 51 estudos sobre a difusão de diferentes tecnologias de saúde, classifica as diversas variáveis em cinco categorias de determinantes poder de compra, necessidades dos pacientes, demanda dos médicos, regulação governamental e métodos de pagamento - além de destacar o impacto de cada uma dessas categorias no processo de difusão tecnológica. Segundo os autores, as necessidades dos pacientes e as demandas dos médicos são fatores que predispõem a incorporação de novas tecnologias, na medida em que fornecem a motivação básica para sua adoção. O poder de compra é visto como um fator que possibilita tornar a motivação uma realidade, determinando em que medida as necessidades dos pacientes e as demandas dos médicos podem ser atendidas. Por fim, os métodos de pagamento e a regulação governamental são fatores que fornecem os incentivos necessários para manter ou desacelerar um determinado nível de difusão tecnológica. Esse modelo foi aplicado pelos 
autores para analisar a difusão de aparelhos de tomografia computadorizada e de ressonância magnética em 30 países da Organização para Cooperação e Desenvolvimento Econômico (OCDE), com dados para o ano 2000, mediante análise estatística de regressão múltipla. O principal achado desse estudo é que a difusão desse tipo de tecnologia é fortemente influenciada por duas categorias de variáveis: poder de compra, expresso pelo gasto total em saúde por habitante/ano, e métodos de pagamento flexíveis, como a remuneração por ato ou procedimento ( $f e e$ for service).

Além da natureza geral do processo de difusão de tecnologias, existem aspectos específicos das tecnologias e dos sistemas de saúde que devem ser considerados. Esses aspectos incluem, entre outros: ${ }^{4}$ agendas de políticas conflitantes (por exemplo, falta de convergência entre a política de saúde e a política de ciência e tecnologia); o volume de novas tecnologias que chegam no mercado (estimativas sugerem que entre 5000 e 8000 novas tecnologias em saúde são lançadas a cada ano nos EUA); a disponibilidade de evidência científica (muitas vezes insuficiente e de qualidade questionável, além da forma peculiar de como ela é interpretada pelas partes interessadas, resultando em dilemas que precisam ser administrados politicamente); os impactos decorrentes da utilização da tecnologia (desperdício de recursos e ineficiências); os custos de desenvolvimento das inovações (necessidade de recuperar o valor investido pelas empresas fabricantes da tecnologia); a estrutura do sistema de saúde (maior ou menor autonomia dada aos prestadores de serviços de saúde para tomar decisões relativas à incorporação de tecnologias); e tendências internacionais (o processo de difusão de tecnologias cruza fronteiras e exerce pressão contínua sobre os gestores de saúde).

Diante dessas considerações, o presente texto apresenta e analisa resultados de um estudo recente, de natureza qualitativa, sobre os determinantes do processo decisório na incorporação do aparelho de tomografia computadorizada por emissão de pósitrons (PET/CT) em quatro estabelecimentos prestadores de serviços de saúde no Brasil, assim como sua relação com empresas operadoras de planos de saúde, que são as instâncias responsáveis pela remuneração da maioria dos procedimentos desses prestadores. Discute-se em que medida os fatores críticos identificados na literatura influenciam as decisões tomadas por dirigentes de estabelecimentos de saúde que, de forma pioneira, incorporam esse tipo de tecnologia no Brasil. A tecnologia selecionada para explorar as questões da investigação foi a tomografia computadorizada por emissão de pósitrons, por reunir um conjunto de atri-butos que tornam mais complexa a decisão relativa à sua incorporação - alto custo e complexidade tecnológica, dependente da disponibilidade de insumos radioativos, com indicações para avaliação e acompanhamento de pacientes oncológicos e alvo de demanda crescente de médicos e pacientes.

No Brasil, o primeiro equipamento de PET/CT foi instalado no Instituto do Coração do Hospital das Clínicas da Faculdade de Medicina da Universidade de São Paulo (Incor), em 2002. Alguns meses depois, outros aparelhos foram comprados e instalados em estabelecimentos de saúde privados da cidade de São Paulo, que já contava com quatro equipamentos disponíveis no final de 2003. A difusão da tecnologia continuou nos anos seguintes, quando passou a ser incorporada também por estabelecimentos de saúde localizados em outras regiões do país. Mais recentemente, dois importantes centros de referência na área de oncologia no país - o Instituto do Câncer do Estado de São Paulo (ICESP) e o Instituto Nacional do Câncer (INCA) - anunciaram a aquisição de aparelhos de PET/CT, sinalizando que a tecnologia também passou a se difundir para dentro do sistema público de saúde.

Apesar do número crescente de equipamentos instalados em instituições públicas e privadas, procedimentos realizados com a tecnologia PET ainda não fazem parte das tabelas de reembolso do Sistema Único de Saúde (SUS). No âmbito da saúde suplementar, o PET foi incluído na nova versão do rol de procedimentos e eventos da ANS, válida a partir de junho de 2010, mas somente duas aplicações estão previstas: a) câncer pulmonar de células não pequenas para caracterização de lesões e estadiamento; e b) linfoma para estadiamento, avaliação da resposta terapêutica e monitoramento da recidiva. Destaque-se, porém, que procedimentos realizados com equipamentos PET-dedicados e PET/CT fazem parte da tabela de Classificação Brasileira Hierarquizada de Procedimentos Médicos (CBHPM), da Associação Médica Brasileira (AMB), desde setembro de 2005. De acordo com a Sociedade Brasileira de Biologia, Medicina Nuclear e Imagem Molecular (SBBMN), as restrições de cobertura do PET nos planos de saúde "atendem a uma preocupação das operadoras, que temem grande impacto nos custos". 11

\section{Métodos}

O trabalho empírico foi realizado por meio de entrevistas em profundidade com representantes de estabelecimentos prestadores de serviços de saúde e de 
operadoras de planos privados de assistência à saúde, no período 2007-2008 (Tabela 1). Para essa finalidade, foram elaborados roteiros de entrevista contendo questões agrupadas em seis blocos: a) identificação do respondente; b) caracterização da atividade; c) fluxo de incorporação de novas tecnologias dentro da instituição; d) incorporação do $\mathrm{PET} / \mathrm{CT}$; e) produção de serviços e perfil da clientela; e f) contratualização prestador x operadora. Em todos os casos, foi realizado contato por telefone e/ou correio eletrônico para agendamento prévio da entrevista. As entrevistas foram realizadas pelos autores, de forma presencial, com um ou mais representantes de cada instituição, e gravadas com o consentimento dos entrevistados. Com o intuito de focar os pontos para discussão, as questões foram enviadas com antecedência para os entrevistados, geralmente uma semana antes da data de realização da entrevista. No total, foram entrevistados seis representantes de prestadores de serviços de saúde e três representantes de operadoras de planos de saúde.

As entrevistas foram transcritas e depois

Tabela 1

Características das instituições selecionadas para o estudo. Brasil, 2007-2008.

\begin{tabular}{|c|c|}
\hline Instituição & Características \\
\hline Prestador 1 & $\begin{array}{l}\text { - Hospital geral, privado filantrópico } \\
\text { - Localização: São Paulo, SP } \\
\text { - } 2 \text { aparelhos de PET/CT } \\
\text { - Data de incorporação: 05/2003 (primeiro aparelho) }\end{array}$ \\
\hline Prestador 2 & $\begin{array}{l}\text { - Hospital geral, privado lucrativo } \\
\text { - Localização: Rio de Janeiro, RJ } \\
\text { - } 1 \text { aparelho de PET/CT } \\
\text { - Data de incorporação: 02/2004 }\end{array}$ \\
\hline Prestador 3 & $\begin{array}{l}\text { - Clínica especializada, privado lucrativo } \\
\text { - Localização: Brasília, DF } \\
\text { - } 1 \text { aparelho de PET/CT } \\
\text { - Data de incorporação: 06/2006 }\end{array}$ \\
\hline Prestador 4 & $\begin{array}{l}\text { - Hospital geral, privado filantrópico } \\
\text { - Localização: Salvador, BA } \\
\text { - } 1 \text { aparelho de PET/CT } \\
\text { - Data de incorporação: } 12 / 2006\end{array}$ \\
\hline Operadora 1 & $\begin{array}{l}\text { - Modalidade: autogestão } \\
\text { - Tamanho aproximado da carteira: } 155 \text { mil vidas } \\
\text { - Tipo de atuação: } 95 \% \text { dos atendimentos concentrados no Estado de SP } \\
\text { - Rede própria: não possui } \\
\text { - Exame de PET/CT: coberto }\end{array}$ \\
\hline Operadora 2 & $\begin{array}{l}\text { - Modalidade: cooperativa médica } \\
\text { - Tamanho aproximado da carteira: } 745 \text { mil vidas } \\
\text { - Tipo de atuação: concentrado na RM de Belo Horizonte; } \\
\text { - Rede própria: } 2 \text { pronto-atendimentos, } 1 \text { maternidade e } 4 \text { centros e núcleos de promoção e } \\
\text { atenção à saúde } \\
\text { - Exame de PET/CT: não coberto }\end{array}$ \\
\hline Operadora 3 & $\begin{array}{l}\text { - Modalidade: medicina de grupo } \\
\text { - Tamanho aproximado da carteira: } 1,8 \text { milhões de vidas } \\
\text { - Tipo de atuação: atendimento nacional, com atuação direta em São Paulo, Grande SP, } \\
\text { Baixada Santista, Jundiaí, Sorocaba, Rio de Janeiro, Minas Gerais e região Nordeste } \\
\text { - Rede própria: } 7 \text { hospitais e } 83 \text { centros clínicos } \\
\text { - Exame de PET/CT: não coberto }\end{array}$ \\
\hline
\end{tabular}

$\mathrm{PET} / \mathrm{CT}=$ tomografia computadorizada por emissão de pósitrons. 
analisadas aplicando as técnicas de análise de conteúdo, 12 com apoio do software NVivo, um programa de computador orientado para o auxílio na análise de dados qualitativos. Uma estratégia de codificação mista foi utilizada: um conjunto prédeterminado de códigos foram traçados a partir do quadro conceitual (determinantes, agentes, relações) e outros códigos surgiram empiricamente a partir dos dados coletados. Primeiro foi analisado o material pertinente a cada caso de forma independente (análise intracaso), o que permitiu identificar a situação geral. Tabelas comparativas foram criadas a fim de condensar o material empírico e identificar padrões recorrentes entre os casos. Isso permitiu explorar em que medida as premissas sociais dos respondentes desempenhavam papel central na determinação da incorporação da tecnologia estudada. Além disso, conforme recomendado pela análise simétrica de controvérsias, 13 foram suspensos a priori todo os julgamentos relacionados ao que é certo ou errado. Assim foi possível explorar, em maior profundidade, os fatores que influenciam a tomada de decisão para cada respondente.

A seleção dos prestadores de serviços de saúde foi realizada a partir de dois critérios. O primeiro diz respeito à localização regional da instituição e o segundo está relacionado com o pioneirismo na incorporação da tecnologia estudada. Dessa forma, a investigação priorizou a seleção de prestadores localizados em diferentes regiões e unidades da federação, assim como o pioneirismo na incorporação do exame de PET/CT. Após contato inicial com instituições que satisfaziam os critérios de seleção, quatro estabelecimentos de saúde concordaram em participar do estudo. Esses estabelecimentos incluem três hospitais gerais e uma clínica especializada. Todos os estabelecimentos de saúde visitados são instituições privadas, sendo que dois deles possuem certificado de entidade filantrópica, ou seja, prestam serviços para o Sistema Único de Saúde. Apesar disso, a tecnologia em questão estava disponível somente para clientela privada. Observase ainda que o aparelho de PET/CT foi incorporado nesses estabelecimentos em diferentes momentos no período que vai de maio de 2003 até dezembro de 2006.

No que diz respeito à seleção das operadoras de planos de saúde, informações obtidas com especialistas indicavam que um número bastante reduzido de operadoras havia incorporado o exame de $\mathrm{PET} / \mathrm{CT}$ no rol de procedimentos assistenciais a serem cobertos. Em virtude dessa limitação, os critérios utilizados para selecionar essas instituições foram seu porte, medido em número de benefi- ciários, e sua modalidade, de acordo com a definição utilizada pela ANS para classificar os diferentes tipos de operadoras médico-hospitalares. Após contato inicial com operadoras que satisfaziam os critérios de seleção, representantes de três operadoras concordaram em participar da investigação. A primeira operadora é da modalidade autogestão, tem foco de atuação regional (SP), não possui rede própria e conta com uma carteira de aproximadamente 155 mil vidas. A segunda é uma cooperativa médica, também possui atuação regional (MG), uma carteira de aproximadamente 750 mil vidas e rede própria. Por fim, a terceira operadora, da modalidade medicina de grupo, possui uma carteira de cerca de 1,8 milhões de vidas, atendimento nacional e rede própria formada por 7 hospitais e 83 centros clínicos, distribuídos nas principais regiões onde sua atuação é direta. Apenas uma das operadoras cobria o exame de $\mathrm{PET} / \mathrm{CT}$ à época da realização da pesquisa de campo.

\section{Resultados}

Os estabelecimentos prestadores de serviços de saúde e as empresas operadoras de planos privados de saúde possuem visões e interesses conflitantes no que se refere à aquisição de novas tecnologias médicas. Enquanto os primeiros adotam uma postura favorável à introdução de inovações tecnológicas, os últimos tendem a ser mais críticos e resistentes. Essa posição divergente encontra uma primeira explicação no papel que as novas tecnologias desempenham nos dois tipos de instituição. Para os prestadores de serviços de saúde, a oferta de um novo procedimento médico, tecnologicamente sofisticado, pode aumentar sua capacidade de gerar receita adicional, assim como elevar seu prestígio junto ao corpo clínico, os pacientes e a concorrência. Para as operadoras de planos de saúde, a introdução de novas tecnologias implicam, num primeiro momento, aumento dos custos assistenciais e, portanto, da taxa de sinistralidade, comprometendo seu desempenho financeiro no curto prazo.

$\mathrm{Na}$ visão dos prestadores, o alto custo de remuneração de alguns procedimentos, associado a uma abordagem de curto prazo, que não considera os benefícios futuros que a tecnologia pode trazer para o paciente, incluindo as economias daí decorrentes, é a principal razão pela qual as operadoras decidem não incorporar esses procedimentos. As operadoras, por sua vez, tendem a olhar o processo acelerado de aquisição de novas tecnologias dentro dos prestadores de serviços de saúde com desconfiança, não apenas pelo impacto financeiro causado pela adoção 
dessas tecnologias, mas também pela falta de evidências científicas que suportem sua aplicação.

O processo de incorporação de inovações tecnológicas faz com que a relação entre esses dois atores se torne ainda mais tensa, pois além da necessidade de negociar o repasse do aumento de custos da assistência médica, a introdução de uma nova tecnologia torna necessário negociar também a remuneração dessa tecnologia. Os relatos das entrevistas mostram que os resultados dessas negociações dependem de vários aspectos, entre os quais cabe destacar: a) o prestígio do estabelecimento prestador de serviços de saúde na percepção da clientela atendida pela operadora; b) o poder de monopólio do prestador em sua área de abrangência; c) a posição ocupada pela operadora no mercado de planos de saúde; e d) a representatividade da operadora em termos de fluxo de atendimento para o prestador.

Os depoimentos sugerem que o processo decisório para aquisição de novas tecnologias médicas nos estabelecimentos de saúde é influenciado tanto por fatores internos quanto externos. Entre os fatores internos, destacam-se os seguintes aspectos: o histórico de pioneirismo da instituição na incorporação de novas tecnologias em sua área de atuação e jurisdição; a pressão do corpo clínico vinculado à instituição, que demanda a aquisição de inovações tecnológicas em suas respectivas especialidades; o prestígio do médico ou da área que solicita a aquisição da tecnologia junto aos dirigentes da instituição; a disponibilidade de recursos financeiros para realização do investimento e para cobrir os custos relacionados à sua operação; e o acesso facilitado à tecnologia, seja por intermédio de instituições parceiras que já possuem a tecnologia, seja mediante a adoção de estratégias de parceria com o fabricante. Os fatores externos incluem a concorrência entre os prestadores de serviços de saúde na busca por maior competitividade e mercado, a existência de evidências de que a tecnologia é segura, eficaz, e o retorno financeiro associado à aquisição da tecnologia.

Em virtude das características de cada prestador, o processo decisório pode envolver a participação de vários profissionais da área técnica e administrativa, incluindo diretoria e conselho administrativo, ou depender única e exclusivamente da vontade do dono da instituição. Vale dizer, o processo de tomada de decisão varia em função do nível de centralização do poder dentro de cada estabelecimento. Entretanto, um aspecto comum deve ser destacado: independentemente da forma como as decisões são tomadas, quem toma a decisão é quase sempre um profissional médico. De fato, os médicos ocupam posição chave no processo de incorporação de novas tecnologias, atuando em, pelo menos, três frentes cruciais: a) solicitando a utilização da tecnologia; b) manipulando e/ou interpretando os resultados obtidos com o uso da tecnologia; e c) decidindo sobre sua aquisição dentro dos estabelecimentos de saúde. No caso da tecnologia estudada, o médico que solicita um exame de PET/CT é, na maioria das vezes, um oncologista, o médico que manipula o equipamento e interpreta os resultados do exame é um médico nuclear (com apoio de um radiologista) e o médico que decide sobre sua incorporação pode ser de qualquer especialidade, desde que ocupe posição de liderança na instituição.

O tipo de relação que os prestadores mantém com os fabricantes da tecnologia também depende da estratégia de atuação de cada estabelecimento. De modo geral, dois tipos de relação foram identificados. O primeiro tipo é uma relação de parceria de longo prazo, no qual o prestador se compromete a atuar como vitrine dos produtos lançados pelo fabricante, recebendo em troca tratamento diferenciado. Por exemplo, o fabricante pode oferecer ao prestador preço mais baixo na aquisição de certos produtos, atendimento exclusivo em serviços de pós-venda, contratos mais vantajosos de manutenção. Como resultado, o prestador conta com um parque instalado com predomínio de equipamentos do mesmo fabricante. O segundo tipo de relacionamento é caracterizado por maior autonomia do prestador frente à atuação do fabricante, em que fidelidade à marca não constitui um aspecto a ser valorizado. Ao contrário, as decisões são tomadas caso a caso, embora a preferência por determinada marca seja, mesmo nesse caso, influenciada pelo histórico de uso de equipamentos de um mesmo fabricante. Nesse segundo tipo de relacionamento, o prestador tende a possuir equipamentos de diferentes fabricantes, numa estratégia que tende a valorizar mais a independência da instituição e a oportunidade comercial de cada momento.

A flexibilização do monopólio na produção e comercialização de radioisótopos de meia-vida curta e os problemas ocasionados pelas dificuldades de fornecimento do insumo necessário à operacionalização do aparelho de PET/CT fizeram com que os prestadores adotassem estratégias específicas para diminuir a dependência em relação à disponibilidade desse insumo. Três estratégias foram identificadas: a) estabelecimento de parcerias com outras instituições para a produção e o fornecimento do FDG18 ; b) montagem de uma estrutura (equipamentos, instalações e pessoal) para produção do insumo dentro da própria instituição; e c) não fazer nada, ou seja, esperar o mercado se ajustar. Embora todos os 
entrevistados reconheçam o profissionalismo dos institutos públicos que produzem e comercializam o radiofármaco, eles também acreditam que a participação da iniciativa privada representa um aspecto positivo, na medida em que possibilita aumentar a oferta desse insumo no mercado e, como consequência, reduzir seu preço de venda.

A introdução de uma tecnologia sofisticada como o equipamento de PET-CT implica mudanças também para a gestão do cuidado, na medida em que sua operacionalização é caracterizada pela necessidade de oferecer um tratamento diferenciado aos pacientes. Em primeiro lugar, pelo fato de que muitas vezes o diagnóstico e o tratamento de tumores requer a participação de médicos de várias especialidades, a tecnologia favoreceu o cuidado interdisciplinar, ou seja, fez com que houvesse maior proximidade entre os profissionais das diferentes especialidades que precisam lidar com o paciente. Em segundo lugar, como o exame de PET-CT requer um preparo prévio do paciente, que necessita estar num ambiente de pouco estímulo e se movimentar o menos possível, antes e depois da injeção do radiofármaco, a tecnologia demandou a construção de uma estrutura dedicada para esse tipo de paciente, incluindo sala de espera e de preparo. Em terceiro lugar, pelas características do paciente que necessita fazer o exame (paciente oncológico, já debilitado e fragilizado emocionalmente), a atenção dispensada pelos profissionais envolvidos na realização do exame tende a ser maior.

Um resumo dos aspectos relacionados às experiências de incorporação da tecnologia $\mathrm{PET/CT}$ entre os prestadores visitados pode ser visto na Tabela 2 .

No que diz respeito à cobertura assistencial oferecida pelas operadoras de planos de saúde, existe consenso de que o rol de procedimentos e eventos da ANS representa a cobertura mínima a ser oferecida aos beneficiários. Logo, a questão que se coloca é a seguinte: "Qual é a estratégia adotada pelas operadoras para os procedimentos e eventos não incluídos no rol da ANS?" Os relatos obtidos permitem identificar a adoção de três estratégias diferenciadas no que diz respeito a essa questão. Uma das estratégias consiste em adotar como referência de cobertura assistencial os procedimentos listados na Classificação Brasileira Hierarquizada de Procedimentos Médicos (CBHPM), que tende a ser mais abrangente que o rol de procedimentos da ANS. Dessa forma, a inclusão de um procedimento novo na CBHPM, como é o caso do exame de PET/CT, implica automaticamente a incorporação desse procedimento na lista de procedimentos a serem remunerados pela operadora. A segunda estratégia contempla a formação de um grupo interno de avaliação de tecnologias em saúde, responsável por elaborar análises de novas tecnologias e pareceres técnicos referentes à sua incorporação na operadora, com base em evidências científicas disponíveis na literatura. A última estratégia consiste em seguir somente os procedimentos e eventos listados no rol da ANS, impedindo a incorporação de tecnologias que não tenham sido previamente incluídas. A primeira estratégia, mais pródiga, é seguida pela operadora de autogestão; a segunda estratégia, mais cautelosa, é adotada pela cooperativa médica; a terceira estratégia, centrada no controle de custos, é seguida pela operadora de medicina de grupo.

Como o rol de procedimentos e eventos da ANS não especifica os valores a serem pagos pelas operadoras, essas últimas adotam diferentes estratégias para construir suas tabelas de remuneração, cujos valores são negociados diretamente com os prestadores de serviços. Duas das três operadoras incluídas no estudo tendem a negociar os valores de todos os procedimentos (honorários, materiais, exames, diária), enquanto a outra operadora, que segue a CBHPM, negocia principalmente as diárias de internação, uma vez que os valores dos procedimentos já estão discriminados na tabela de referência utilizada por ela. Essa negociação, por sua vez, leva em consideração diferentes aspectos, como o perfil do prestador (público atendido, padrão de hotelaria, complexidade tecnológica instalada, poder de monopólio em sua área de abrangência) e o volume de negócio que a operadora representa para o prestador. Como resultado, as operadoras adotam diferentes tabelas de remuneração, uma para cada perfil de prestador, sendo que essas tabelas refletem, em última instância, o poder de negociação das operadoras com os prestadores.

Nosso estudo identificou que diversos mecanismos são utilizados pelas operadoras de planos de saúde no sentido de influenciar a incorporação de tecnologias que não estão incluídas no rol de procedimentos e eventos em saúde da ANS. Os principais mecanismos adotados, de acordo com os entrevistados, são os seguintes: apresentar conhecimento técnico-científico desfavorável à incorporação; possuir profissionais que trabalham com exclusividade para a operadora; possuir rede própria de prestadores de serviços; trabalhar somente com equipes de profissionais que não utilizam a tecnologia; não contratar e/ou recusar a remunerar o procedimento; e comercializar planos mais básicos, chamados de "chão de fábrica".

Algumas fragilidades ou desafios do sistema de saúde suplementar foram apontados pelos represen- 


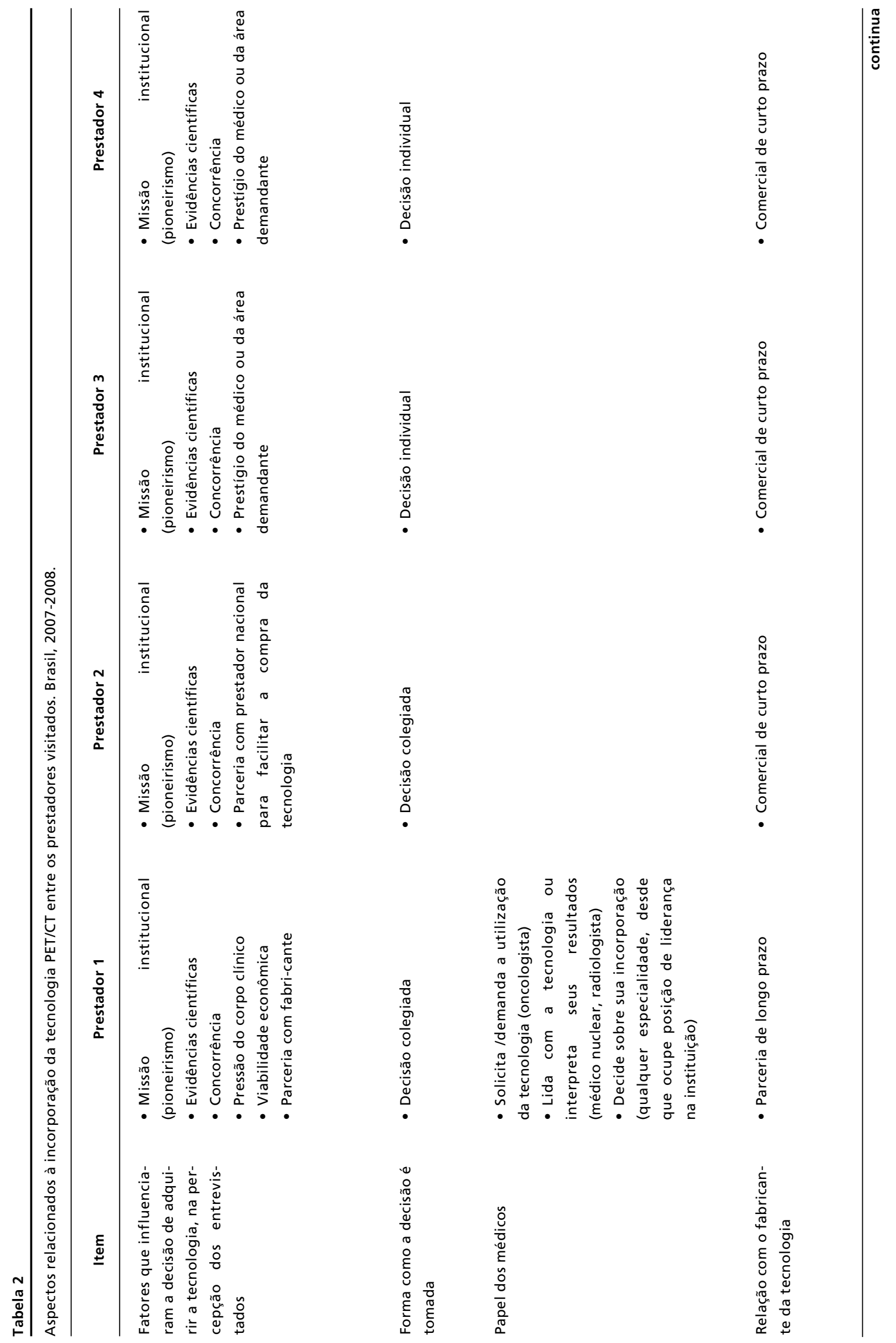




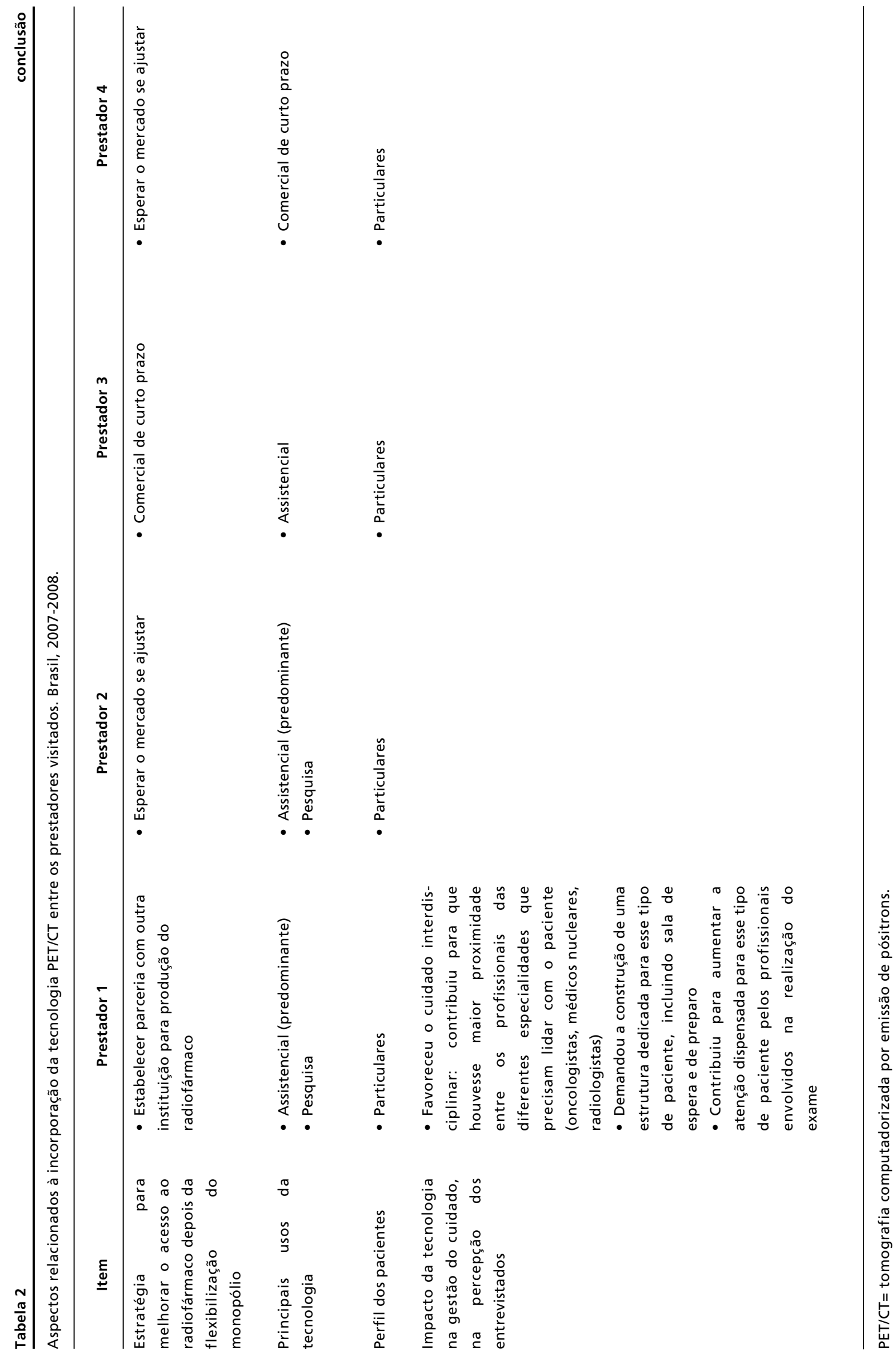


tantes das operadoras, que expressaram preocupação quanta à sustentabilidade de suas operações. A quantidade de procedimentos realizados no âmbito da saúde suplementar, muitas vezes sem necessidade, e a forma de remuneração predominante (por procedimento) representam dois problemas que oneram as operadoras e tornam o sistema pouco eficiente. A dinâmica interna do mercado de saúde suplementar também foi objeto de preocupação das operadoras, que indicaram a existência de um processo "autofágico", no qual as operadoras maiores tendem a adquirir aquelas que apresentam dificuldades de permanecer no mercado, implicando maior centralização de capital nesse segmento. Os entrevistados destacaram também que a dinâmica econômica tende a afetar o funcionamento das operadoras em dois aspectos diferentes. O primeiro é a constatação de que as baixas taxas de crescimento da economia brasileira não favorecem a expansão do mercado. $\mathrm{O}$ segundo é que o processo de fusões e aquisições não é exclusivo do mercado de saúde suplementar e acontece de uma maneira geral, em todos os setores, aumentando o poder de negociação das empresas que são clientes das operadoras (planos coletivos). Por fim, no que diz respeito à incorporação de novas tecnologias, foi mencionado que as operadoras enfrentam uma situação particularmente difícil, na medida em que elas são pressionadas tanto por aqueles que geram receita (beneficiários), quanto por aqueles que representam despesa assistencial (prestadores de serviços de saúde). Nos dois casos, a pressão é para que novos procedimentos sejam incluídos nas tabelas de remuneração das operadoras.

A Tabela 3 sintetiza os aspectos relacionados à inserção das operadoras de planos de saúde no processo de incorporação de novas tecnologias médicas.

Tabela 3

Aspectos relacionados à inserção das operadoras de planos de saúde no processo de incorporação de novas tecnologias. Brasil, $2007-2008$.

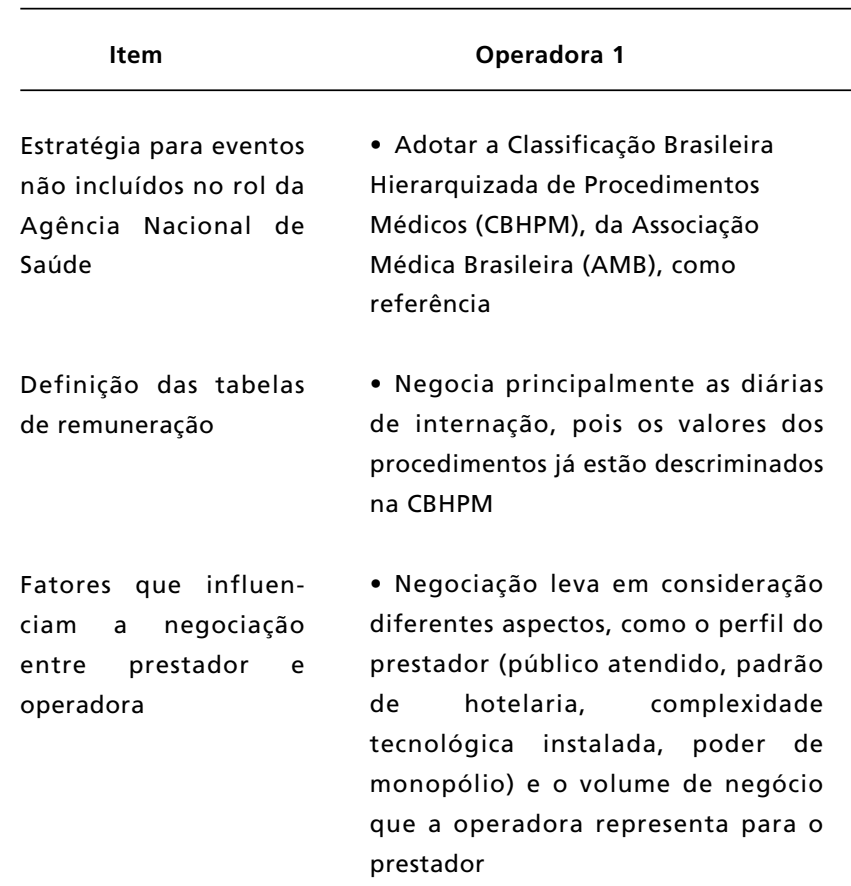

Operadora 2

Operadora 3

- Formar um grupo interno para avaliação de tecnologias, com o objetivo de elaborar análises de novas tecnologias e pareceres técnicos sobre sua incorporação

- Negocia os valores de todos os procedimentos (honorários, materiais, exames, diárias de internação)
- Impedir a incorporação de todo e qualquer procedimento não listado no rol da ANS

- Negocia os valores de todos os procedimentos (honorários, materiais, exames, diárias de internação) 
Aspectos relacionados à inserção das operadoras de planos de saúde no processo de incorporação de novas tecnologias. Brasil, $2007-2008$.

\begin{tabular}{|c|c|c|c|}
\hline Item & Operadora 1 & Operadora 2 & Operadora 3 \\
\hline Dificuldades relatadas & $\begin{array}{l}\text { - Pressão de prestadores e usuários } \\
\text { pela inclusão e cobertura de novas } \\
\text { tecnologias de remuneração dos } \\
\text { - Forma de } \\
\text { procedimentos (fee for service), } \\
\text { indutora de demanda } \\
\text { - Quantidade de procedimentos } \\
\text { desnecessários realizados no âmbito } \\
\text { da saúde suplementar } \\
\text { - Estrutura oligopólica do mercado } \\
\text { de saúde suplementar em algumas } \\
\text { cidades e regiões, implicando } \\
\text { barreiras à entrada e baixa } \\
\text { competitividade } \\
\text { - Dinâmica da economia brasileira, } \\
\text { que não favorece a expansão do } \\
\text { mercado Processo de fusões e aquisições em } \\
\text { outros mercados, aumentando o } \\
\text { poder de negociação das empresas } \\
\text { que são clientes das operadoras } \\
\text { (planos coletivos) }\end{array}$ & $\begin{array}{l}\text { - Possuir profissionais que } \\
\text { trabalham com exclusividade } \\
\text { - Trabalhar somente com } \\
\text { equipes de profissionais que não } \\
\text { utilizam a tecnologia } \\
\text { - Possuir rede própria de } \\
\text { prestadores de serviços } \\
\text { - Não contratar o procedimento } \\
\text { / recusar a remunerar }\end{array}$ & $\begin{array}{l}\text { - Trabalhar somente com } \\
\text { equipes de profissionais que } \\
\text { não utilizam a tecnologia } \\
\text { - Possuir rede própria de } \\
\text { prestadores de serviços } \\
\text { - Comercializar planos mais } \\
\text { básicos } \\
\text { - Não contratar o } \\
\text { procedimento / recusar a } \\
\text { remunerar }\end{array}$ \\
\hline
\end{tabular}

CBHPM= Classificação Brasileira Hierarquizada de Procedimentos Médicos; ANS = Agência Nacional de Saúde.

\section{Discussão}

Nosso estudo sugere que a aquisição de equipamentos médicos de alto custo e densidade tecnológica, como é o caso da tecnologia PET, não é comandada por formuladores de políticas de saúde nem por terceiros pagadores dos setores público (SUS) e privado (planos de saúde). Ao contrário, as decisões são tomadas por gestores de hospitais e clínicas que possuem condições de mobilizar os recursos necessários (poder de compra) para adquirir esse tipo de tecnologia. Essas decisões, por sua vez, são fortemente influenciadas pela atuação dos médicos e dos representantes dos fabricantes do equipamento, que atuam como agentes de mudança que exercem pressão sobre a direção do hospital. De acordo com Rogers, ${ }^{8}$ um agente de mudança é um indivíduo que influencia as decisões de terceiros numa direção desejada por ele. No caso do representante da indústria produtora de equipamentos médicos, seu trabalho é influenciar as decisões dos médicos, que são os usuários primários de seus produtos, mediante a oferta de material educativo, recursos para pesquisas e outros tipos de incentivo, num esforço para convencê-los de que seus produtos são melhores do que os da concorrência. Os médicos, por sua vez, representam a ligação entre os fabricantes da tecnologia, os estabelecimentos de saúde e os pacientes, exercendo pressão junto aos tomadores de decisão para que determinada 
tecnologia seja adquirida.

Como as decisões para incorporar esse tipo de tecnologia são tomadas principalmente por dirigentes dos estabelecimentos de saúde, é importante identificar quais fatores influenciam essas decisões no nível organizacional. A literatura ${ }^{14-15}$ registra a existência de três explicações ou modelos de tomada de decisão: a) o modelo de gestão fiscal, que prioriza a maximização dos lucros; b) o modelo institucional estratégico, que enfatiza a proeminência tecnológica; e c) o modelo médico-individualista, focalizado na excelência clínica. A primeira explicação relaciona o comportamento organizacional aos retornos financeiros antecipados, de modo que uma tecnologia nova e custosa será incorporada somente se o investimento for capaz de maximizar os lucros do estabelecimento de saúde. A segunda explicação associa tecnologias novas, caras e sofisticadas com a qualidade da assistência, o que é usado pelo hospital para promover sua imagem como líder na incorporação de tecnologias, atraindo assim mais médicos e pacientes, além de satisfazer os desejos dos dirigentes de administrar uma instituição atualizada, de alta qualidade e prestígio, e os desejos dos médicos de trabalhar num local equipado com as tecnologias mais recentes. A terceira explicação assume que os estabelecimentos de saúde atuam como agentes que defendem os interesses de seus pacientes, e que os médicos tomam decisões com base no melhor julgamento das necessidades clínicas do paciente, de modo que a probabilidade de um hospital que possui necessidades clínicas mais elevadas adotar novas tecnologias médicas é maior do que a de outro hospital, mesmo se as condições financeiras, competitivas ou de prestígio sugerem a adoção de ações alternativas. Convém destacar que esses três modelos de tomada de decisão não são mutuamente exclusivos, mas complementares. De fato, a incorporação de novas tecnologias médicas é um processo complexo e todas as três explicações foram relatadas como razões importantes para a aquisição do aparelho de PET/CT. Entretanto, com base nas informações coletadas nas entrevistas, podemos afirmar que as três razões não possuem o mesmo nível de importância no processo de tomada de decisão. Embora a viabilidade econômica do investimento e o perfil clínico dos pacientes sejam aspectos relevantes, nosso estudo sugere que esses critérios não são os principais determinantes do processo decisório. Ao contrário, uma estratégia de diferenciação baseada em liderança tecnológica foi relatada como o determinante mais forte para a aquisição da tecnologia estudada. Destaque-se que essa explicação é consistente com os resultados apresentados por Teplensky et al.,15 em um estudo sobre adoção de aparelhos de ressonância magnética em mais de 500 hospitais nos EUA.

A forma como o sistema de saúde está estruturado no Brasil representa uma explicação para o papel proeminente desempenhado pelos estabelecimentos de saúde privados no processo decisório. Como é sabido, o sistema de saúde brasileiro é formado por dois subsistemas paralelos - e muitas vezes sobrepostos: o subsistema público, que oferece cobertura universal para toda a população e é financiado com recursos fiscais pagos por toda a sociedade, e o subsistema privado, de natureza mercantil, que inclui os diversos tipos de seguros e planos de saúde, coletivos e individuais. Com base nos cinco tipos de sistemas de saúde sugeridos por Propper e Green,16 que considera a combinação público-privada de prestação de serviços de saúde e de financiamento, podemos dizer que o Brasil possui um sistema de saúde misto, com segmentação de clientela e múltiplas relações envolvendo o público e o privado. Em termos de provisão de serviços, o subsistema público compreende a maior rede de estabelecimentos de atenção primária em saúde, ao passo que a maioria das instituições secundárias e terciárias (hospitais e clínicas) é de natureza privada. A proporção de médicos no subsistema privado é maior do que no subsistema público, mas o inverso ocorre com os profissionais de enfermagem.17 Do ponto de vista financeiro, o sistema de saúde brasileiro apresenta a mesma situação observada em muitos países em desenvolvimento, onde os recursos privados tendem a ser mais expressivos. De fato, os recursos públicos representam somente $41,6 \%$ do gasto total com saúde no Brasil.18 Paradoxalmente, supõe-se que essa pequena proporção seja suficiente para oferecer cobertura para mais de $75 \%$ da população que depende do subsistema público. Ora, como a maior parte do faturamento dos grandes hospitais e clínicas de média e alta complexidade no Brasil provém de pagamentos realizados no âmbito do subsistema privado, via planos de saúde e desembolso direto dos pacientes, as decisões relativas à incorporação de novas tecnologias nesses estabelecimentos são tomadas, na maioria das vezes, de forma autônoma e sem vínculo direto com as prioridades definidas pelos gestores do sistema de saúde.

Embora alguns mecanismos de controle sejam usados pelas autoridades públicas de saúde e agências reguladoras a fim de incentivar ou desincentivar a adoção e o uso de tecnologias na área da saúde, tais como a produção e o uso de estudos de avaliação tecnológica em saúde (ATS), o sistema de registro de produtos da Agência Nacional de Vigilância 
Sanitária (ANVISA) e o manejo das tabelas de remuneração do SUS e do rol de procedimentos e eventos em saúde da ANS, o impacto desses mecanismos no processo decisório dos grandes estabelecimentos de saúde privados tende a ser reduzido, dado que muitos desses hospitais dependem pouco dos recursos do subsistema público. Como resultado, muitas decisões são guiadas por forças do mercado e estratégias institucionais, com controle limitado do poder público. Além disso, como muitos médicos e estabelecimentos de saúde são remunerados em função do seu volume de atendimento e produção de serviços, eles são encorajados a adquirir novas tecnologias e realizar o maior número possível de procedimentos, muitas vezes desnecessários ou de eficácia duvidosa.

\section{Conclusões}

A instituição de uma política nacional de gestão de tecnologias em saúde representa, sem dúvida, uma iniciativa importante no contexto brasileiro, onde os instrumentos disponíveis para controlar o ritmo de difusão de tecnologias caras e complexas parecem não surtir muito efeito nas decisões tomadas por dirigentes de grandes estabelecimentos prestadores de serviços de saúde, principalmente hospitais e clínicas que atuam de forma específica no segmento privado e que possuem maior autonomia em suas decisões de gasto. Apesar do avanço representado por essa iniciativa, nosso estudo aponta a existência de aspectos que tendem a criar obstáculos para o cumprimento do objetivo geral da política, que é o de "maximizar os benefícios de saúde a serem obtidos com os recursos disponíveis, assegurando o acesso da população a tecnologias efetivas e seguras, em condições de equidade". ${ }^{1}$

Em primeiro lugar, porque a gestão da difusão de tecnologias depende de aspectos como as características das tecnologias e dos sistemas de saúde, assim como da disponibilidade de instrumentos de política apropriados. Com relação a esse último aspecto, existe uma grande diversidade de instrumentos que podem ser utilizados para influenciar o ritmo da adoção de novas tecnologias nos sistemas de saúde, seja do lado da oferta (planejamento público e legislação para regular o fornecimento, a distribuição e a difusão de tecnologias, instalações e profissionais), seja do lado da demanda (controle de gasto, formas de reembolso, diretrizes clínicas, informação e conhecimento técnico-científico para os prestadores e o público em geral, incentivos, gestão do desempenho, regulação e auditoria médica). Por isso mesmo, é pouco provável que um único modelo de gestão de tecnologias seja eficaz em todas as situações. Ao contrário, a abordagem a ser utilizada precisa considerar a tecnologia em questão e as particularidades do sistema de saúde, com alguns instrumentos funcionando para algumas tecnologias ou circunstâncias, e não para outras.

Em segundo lugar, a ênfase na adoção de estudos de avaliação de tecnologias em saúde (ATS) pode representar uma estratégia pouco eficaz no contexto brasileiro. Isso porque, dado que muitas forças sociais, econômicas e políticas moldam a difusão e o uso das tecnologias em saúde, esse tipo de abordagem possui impacto limitado na tomada de decisão, especialmente quando os adotantes (hospitais, clínicas) reunem todas as condições necessárias (disponibilidade de recursos financeiros, autonomia para decidir) para efetuar a compra de determinada tecnologia. Além disso, essa abordagem possui o foco direcionado para o exame de fatores vinculados ao produto já acabado, isto é, quando as inovações já estão prontas para serem introduzidas nos sistemas de saúde. Dessa forma, a ATS precisa ser entendida como apenas uma fonte de informação entre outras que são valorizadas e usadas em tomadas de decisão e formulações de políticas.

Em terceiro lugar, o impacto da política nacional de gestão de tecnologias no segmento privado de saúde tende a ser ainda mais incerto, na medida em que suas diretrizes possuem caráter meramente recomendatório nas ações das duas agências reguladoras na área da saúde. Essa limitação significa, na prática, que os instrumentos atualmente administrados por essas agências continuarão funcionando de forma pouco associada com as decisões tomadas no âmbito da política de saúde, contribuindo para a geração de conflitos no âmbito do sistema. Mesmo que não fosse esse o caso, a política ainda teria pouca influência sobre as decisões de gasto no âmbito do segmento privado de saúde, na medida em que esse segmento conta com estabelecimentos capazes de atrair uma clientela particular com elevado poder de compra e disposta a pagar o preço necessário para ter acesso a essas tecnologias.

O poder público brasileiro não pode mais ignorar que uma parcela cada vez maior de tecnologias médicas tem sido incorporada no sistema de saúde via prestadores privados, cuja lógica de atuação não está relacionada com as necessidades de saúde da população ou com as prioridades definidas na política de saúde. Somente a adoção de um conjunto de medidas e políticas públicas capazes de influenciar as decisões de gasto dos agentes privados poderá reverter essa situação, contribuindo para 
equilibrar o desejo profissional e institucional dos médicos e estabelecimentos de saúde de praticar uma

\section{Referências}

1. Brasil. Ministério da Saúde. Portaria 2.690, de 5 de novembro de 2009. Institui, no âmbito do Sistema Único de Saúde (SUS), a Política Nacional de Gestão de Tecnologias em Saúde. Brasília, DF. Diário Oficial da União; 2009.

2. Brasil. Ministério da Saúde. Portaria 2.480/GM de 13 de outubro de 2006. Submete a proposta de Política Nacional de Gestão de Tecnologias em Saúde a Consulta Pública. Brasília, DF. Diário Oficial da União; 2006.

3. OECD. Health technology and decision making. Paris: OECD; 2005.

4. Murtagh J, Foerster V. Managing technology innovation. 2009. [2010 Fev 17]. Disponível em: http://www.cadth.ca/ media/policy_forum_section/Managing_Technology_Diffu sion_e.pdf.

5. Brasil. Agência Nacional de Saúde Suplementar. Resolução Normativa 211, de 12 de janeiro de 2010. Brasil: Diário Oficial da União; 2010

6. Camargo EE. Experiência inicial com PET/CT. Radiol Bras. 2005; 38(1): III-V.

7. Canadian Agency for Drugs and Technologies in Health. FAQ: What is health technology? 2007. [2010 Fev 10] Disponível em: http://www.cadth.ca/index.php/en/hta/faq.

8. Rogers EM. Diffusion of innovations. 4 ed. New York: The Free Press; 1995.

9. Cain M, Mittman R. Diffusion of innovation in healthcare 2002. [2010 jan 22]. Disponível em: http://www.chcf.org/ /media/Files/PDF/D/PDF\%20DiffusionofInnovation.pdf. medicina moderna e custosa num contexto de desigualdades e necessidades básicas em saúde.

10. Oh EH, Imanaka Y, Evans E. Determinant of the diffusion of computed tomography and magnetic resonance imaging. Int J Technol Assess Health Care. 2005; 21: 73-80.

11. SBBMN. Notícias - Boa notícia: PET/CT agora faz parte do Rol de Procedimentos da ANS. 2010. [2010 mar 03]. Disponível em: http://www.sbbmn.org.br/v3/sbbmn.php? modulo=noticias\&id_not $=326$.

12. Bardin L. Análise de conteúdo. Lisboa: Edições 70; 1977.

13. Martin B. Dissent and heresy in medicine: models, methods, and strategies. Soc Sci Med. 2004, 58: 713-25.

14. Greer AL. Adoption of medical technology: the hospital's three decision systems. Int J Technol Assess Health Care. 1985; 1: 669-79.

15. Teplensky JD, Pauly MV, Kimberly JR, Hillman AL, Schwartz JS. Hospital adoption of medical technology: an empirical test of alternative models. Health Serv Res. 1995; 30: 437-65

16. Propper C, Green K. A large role for the private sector in health care? A review of the arguments. 1999. [2010 Jan 23]. Disponível em: http://www.bristol.ac.uk/cmpo/publications/papers/1999/wp9.pdf.

17. IBGE (Instituto Brasileiro de Geografia e Estatística). Pesquisa de assistência médico-santária 2005. Rio de Janeiro; 2005.

18. WHO (World Health Organization). World Health Statistics 2010. Geneve; 2009. [2010 set 3]. Disponível em: $<$ http://www.who.int/whosis/hostat/EN_WHS10_Full.pdf

Recebido em 5 de abril de 2010

Versão final apresentada em 11 de outubro de 2010

Aprovado em 20 de outubro de 2010 